Anne J.A. Drost-Wijnne, Ralph A.C. Van Wezel*, Maarten J. Deenen, Joost P.C.M. Van

Doornmalen Gomez Hoyos and René J.E. Grouls

\title{
A Proof of Principle Study of the Terminal Sterilization of Prefilled Syringes Using A Water Cascade Process
}

https://doi.org/10.1515/pthp-2018-0020

Received May 30, 2018; revised July 19, 2018; accepted July 19, 2018

\section{Abstract}

Background: A new development in drug compounding is the production of ready-to-administer sterilized prefilled syringes. A challenge with these syringes is the method of terminal sterilization. There is no information available whether water cascade sterilization is a suitable method. We investigated the effect of this sterilization method on cyclic olefin (co)polymer (CCP/COC) syringes.

Methods: For two brands ten prefilled syringes were sterilized using water cascade sterilization. The closure integrity, stopper movement, weight, diameter and physical appearance were determined before and after sterilization. As sterility test, additional syringes were filled with tryptic soy broth (TSB) and sterilized. After fourteen days microbiological growth was determined. Results: Closure integrity testing showed no dye penetration inside the syringe. Together with the results for weight this showed that closure integrity is guaranteed. No significant stopper movement, deviation in diameter or visual anomalies were observed. No microbiological growth in TSB was visible.

Conclusions: The results of this proof of principle study show that the physical and microbiological stability of the cyclic olefin (co)polymer syringes is guaranteed

*Corresponding author: Ralph A.C. Van Wezel, Department of Clinical Pharmacy, Catharina Hospital, Eindhoven, Netherlands, E-mail: ralph.v.wezel@catharinaziekenhuis.nl

Anne J.A. Drost-Wijnne, Maarten J. Deenen, Department of Clinical Pharmacy, Catharina Hospital, Eindhoven, Netherlands,

E-mail: anne.wijnne@catharinaziekenhuis.nl; maarten.deenen@catharinaziekenhuis.nl Joost P.C.M. Van Doornmalen Gomez Hoyos, Department of Applied Physics, Eindhoven University of Technology, Eindhoven, Netherlands, E-mail: vdo@lambda-projects.nl

René J.E. Grouls, Department of Clinical Pharmacy, Catharina Hospital, Eindhoven, Netherlands,

E-mail: rene.grouls@catharinaziekenhuis.nl during sterilization using a water cascade sterilizer. These results do not rule out the necessity for further stability experiments (e.g. interaction with drug product) to further proof the concept.

Keywords: ready-to-administer, compounding, sterilization, water cascade sterilization, prefilled syringe

\section{Introduction}

Several studies have shown that medication errors commonly occur during preparation of parenteral medication, including bacterial contamination, wrong dose calculations, wrong dilution and inaccuracy [1-6]. In order to diminish these errors, a shift currently occurs in Dutch hospitals from reconstitution of intravenous drugs by nurses on the ward to centralized reconstitution by pharmacy technicians in the hospital pharmacy.

The latest development is stock production of ready to administer products like aseptic prefilled ready-toadminister syringes. This development is based on professional standards used in the Netherlands such as described in the GMP-Z (Good Manufacturing Practice in hospitals, Z stands for "ziekenhuis", the Dutch word for "hospital"). The GMP-Z is a Dutch derivative from the European Good Manufacturing Practice guidelines with extended principles and guidelines that specifically apply to the aseptic and sterile production of drugs in a hospital pharmacy setting. These addenda include guidelines for the aseptic preparations for an individual patient, but also for larger scale preparation of aseptic prefilled single use polypropylene syringes. When prepared under aseptic conditions, the guideline for the preparation of aseptic prefilled syringes states a shelflife of maximally one month stored at $2-8{ }^{\circ} \mathrm{C}$ due to microbiological stability. In daily practice this leads to high spillage of syringes and high storage costs. These problems can be overcome by introducing terminally sterilized prefilled syringes, made from a type of plastic that is heat resistant $[7,8]$. These can be produced on 
large scale in the hospital pharmacy. Another advantage of sterilized prefilled syringes is an increase in shelf-life and the possibility of storage at room temperature. A challenge to be addressed compounding these prefilled syringes is the method of terminal sterilization. There is only little evidence available in literature that describes the effect of terminal sterilization on (prefilled) ready-toadminister syringes. The studies published describe the use of steam and radiation sterilization [8, 9]. However, to the best of our knowledge no studies have yet described the use of water cascade sterilization. Water cascade sterilization is a commonly used method in Dutch hospital pharmacy departments for the sterilization of parenteral thermostable drug products stored in closed primary packages other than syringes, such as glass and polypropylene containers [10]. With this method of sterilization temperature is gradually increased and counterpressure can be applied to prevent deformation of the containers. Water cascade sterilization is a valid alternative for steam sterilization as long as the conditions that deliver an adequate and reproducible level of lethality are met, as stated in the European Pharmacopoeia [11]. To determine whether this sterilization method is suitable, the physical, microbiological, and chemical (e.g. interaction with drug product) stability must be demonstrated. The aim of this proof of concept study is to investigate the effect of water cascade sterilization on the physical and microbiological stability of sterilized prefilled syringes on small scale. With positive results the effect will be investigated on larger scale with a full validation of this sterilization method for prefilled syringes.

\section{Materials and methods}

In order to demonstrate the physical and microbiological stability of water cascade sterilization two brands of commercially available syringes (A and B) were selected; brand A made of Cyclic Olefin Copolymer (COC) and brand B made of Cyclic Olefin Polymer (COP), both with a butyl rubber tipcap and bromobutyl rubber stoppers. The syringes were packed in nest and tub configuration according to ISO 11040-7 2015. To determine the microbiological stability the closure integrity test, the stopper movement and the sterility test were performed. Stopper movement was calculated to determine if the sterile content of the syringe comes into contact with the nonsterile part of the syringe during sterilization.

\section{Filling process}

For both brand A and B, syringes were filled at the stopper side under GMP conditions (laminar airflow with class $\mathrm{C}$ background). For physical stability testing ten syringes were filled with $50 \mathrm{ml} 0.9 \%$ saline solution (Baxter, Viaflo) and for sterility testing ten syringes were filled with $50 \mathrm{ml}$ Trypton Soya Broth (TSB, BioTrading). The stopper was placed in the syringe using a needle inserted alongside the stopper for de-aeration. A maximum of $0.5 \mathrm{ml}$ of air inclusion was accepted, based on the amount of air to be expected in the automatic filling process. The needle was removed when the stopper was in place. This procedure was used to mimic an automatic filling process.

\section{Sterilization}

All syringes were sterilized using a combined steam and water cascade sterilizer (De Lama DLOV/S-C) with chamber size $1450 \mathrm{~mm}$ (diameter) x $1450 \mathrm{~mm}$ (length). In this study the water cascade process was used. According to the European Pharmacopoeia (Ph. Eur.), the syringes were sterilized at $121^{\circ} \mathrm{C}$ for 15 minutes. The temperature inside the product and inside the chamber is measured at the coolest part of the sterilizer, these parts were established during the general validation process of the sterilizer. Figure 1 depicts the temperature and pressure in the used sterilization process. It consists of four phases (temperature setpoint; pressure setpoint), indicated in Figure 1 with $A-D$, the warm up period, $\mathrm{A}\left(122^{\circ} \mathrm{C}\right.$; $\left.338 \mathrm{kPa}\right)$, the plateau period, $\mathrm{B}\left(122^{\circ} \mathrm{C}\right.$; $330 \mathrm{kPa})$, forced cooling with support pressure, $\mathrm{C}\left(70^{\circ} \mathrm{C}\right.$; $340 \mathrm{kPa})$ and the cooling down period, $\mathrm{D}\left(70^{\circ} \mathrm{C}\right.$; $100 \mathrm{kPa}$ ). The sterilization process is validated with a full load of $50 \mathrm{ml}$ and $100 \mathrm{ml}$ injection vials according to the criteria as described in the ISO 17665 [12]. The nest with syringes, fixated in a mesh sterilization tray with the tipcap facing upward, were placed in the middle of the top shelf of the sterilizer. The syringes were sterilized alongside $100 \mathrm{ml}$ vials to simulate a full sterilization load. The two Pt100 temperature sensors (I and II) 


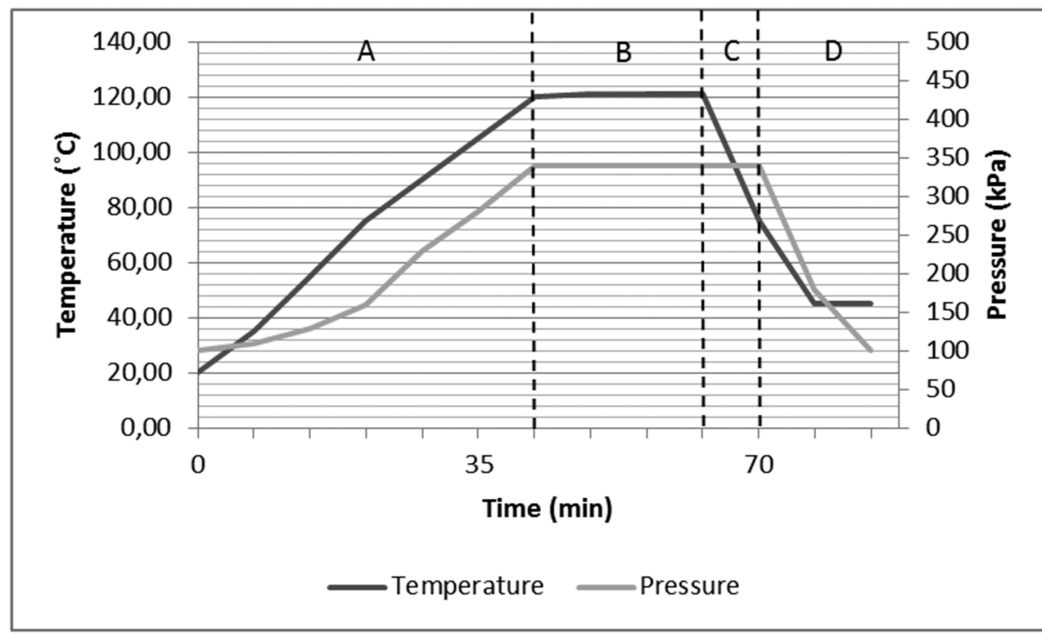

Figure 1: Schematic view of sterilization process. to monitor temperature of the load were each inserted in a $100 \mathrm{ml}$ vial. As a negative control experiment, two prefilled syringes of each brand were not sterilized.

\section{Closure integrity}

The closure integrity was determined using the methylene blue dye ingress test (DIT) as described in the Ph. Eur. (9th ed.) and the technical report of the Parenteral Drug Association (PDA) [13]. The syringes filled with saline solution were immersed in a $1 \mathrm{~g} / \mathrm{L}$ solution of methylene blue with the tipcap facing downward; two not-sterilized syringes with saline solution were included as negative control. The pressure was reduced to $50 \mathrm{kPa}$ atmosphere for 15 minutes, followed by a 15 minute increase in pressure to $200 \mathrm{kPa}$ atmosphere and afterwards the pressure was returned to atmospheric pressure. A pressure container (Walther LDG 20) was used to achieve negative and positive pressure. After immersion the syringes were rinsed with water, dried with paper tissues and visually inspected for the presence of dye. From all immersed syringes a sample was analyzed on a UV spectrophotometer (Shimadzu UV-2401 PC) between 654 and $700 \mathrm{~nm}$. A solution of $0.5 \mu \mathrm{g} / \mathrm{mL}$ methylene blue was used as positive control. The Lower Limit of Quantification (LLOQ) of the UV analysis was $0.8 \mu \mathrm{g} / \mathrm{L}$. All syringes with a calculated concentration below the LLOQ were considered free of methylene blue.

\section{Stopper movement}

Stopper movement was calculated using the equation for linear thermal expansion (eq. (1)) and the combined gas law (eq. (2)). This method is an alternative way to determine stopper movement without the use of intricate physical tests. The sterilizer Pt100 sensors log the temperature (in the two $100 \mathrm{ml}$ vials and chamber) and pressure (in the chamber) every three seconds, this data was used to calculate stopper movement for both syringe brands.

$$
\Delta \boldsymbol{L}=\alpha \boldsymbol{L} \Delta \boldsymbol{T}
$$

Where $\Delta L$ is stopper movement ( $\mathrm{mm}), \alpha$ is linear thermal expansion of water $\left(0.07^{-3} \mathrm{~K}^{-1}\right)$ and $\Delta T$ is the difference in temperature (K).

$$
\frac{P V}{T}=k
$$

Where $P$ is pressure $(\mathrm{kPa}), V$ is volume $\left(\mathrm{mm}^{3}\right), T$ is temperature (K) and $k$ is a constant.

In the calculations it is assumed that:

- The gas and fluid behave as independent systems

- The shape of the stopper equals the shape of the tip of the syringe, therefore the syringe can be seen as a cylinder.

- The gas behaves as an ideal gas; therefore the ideal gas law applies.

- The liquid behaves like water; therefore the thermal expansion coefficient of water can be used. 
- $\quad$ The stopper can move freely, therefore the pressure in the syringe equals the pressure in the chamber.

- The volume changes of the syringe are negligible relative to the volume changes of the gas and liquid.

- The amount of gas present inside the syringe was estimated to be $0.5 \mathrm{ml}$.

\section{Sterility testing}

The syringes filled with TSB were placed in the incubator at $30-35^{\circ} \mathrm{C}$ for 14 days. The syringes were visually inspected for the presence of bacteria by turbidity at 3 and 14 days after sterilization.

\section{Other physical tests}

To investigate the mechanical influence of the sterilization process several measurements on the physical stability were performed including weight, diameter and physical appearance. Weight was measured on a Mettler Toledo XS105 scale $(\mathrm{d}=0.1 \mathrm{mg})$ before sterilization and afterwards when the syringes were completely dry. The diameter of five syringes was measured at three positions (Figure 2) before and after sterilization using an analog Vernier caliper (Nippon Sokutei). The position of measurement was marked to ensure that this was the same before and after sterilization. After sterilization the syringes were visually inspected regarding clarity and color according to Ph. Eur. monographs 2.2.1 and 2.2.2. The syringes, tipcap and stopper were visually inspected to rule out physical deformation.

\section{Statistical analysis}

The skewness and kurtosis of the data were analyzed to determine normal distribution. Diameters before and after sterilization were statistically compared using the paired $t$-test or the non-parametric Wilcoxon Rank test, where appropriate. All statistical tests were conducted using IBM SPSS Statistics version 23.0.

\section{Results}

\section{Closure integrity}

The results for closure integrity are shown in Table 1. The positive control showed maximal absorbance at a wavelength of $664 \mathrm{~nm}$. No absorption at $664 \mathrm{~nm}$ was found in all twelve syringes for both brands, that is, including the two negative controls. On visual inspection of the syringes after dye ingress testing the methylene blue had penetrated between the ribs of the stopper in one sterilized syringe and in one negative control of brand A. However, the syringes that showed dye between the ribs of the stopper showed no UV absorption within the saline solution. Taking into account the LLOQ, this shows that the concentration methylene blue inside the syringes was less than $0.8 \mu \mathrm{g} / \mathrm{L}$.

\section{Stopper movement}

The results for stopper movement are depicted in Figure 3. Figure 3(C) and 3(D) show the volume change

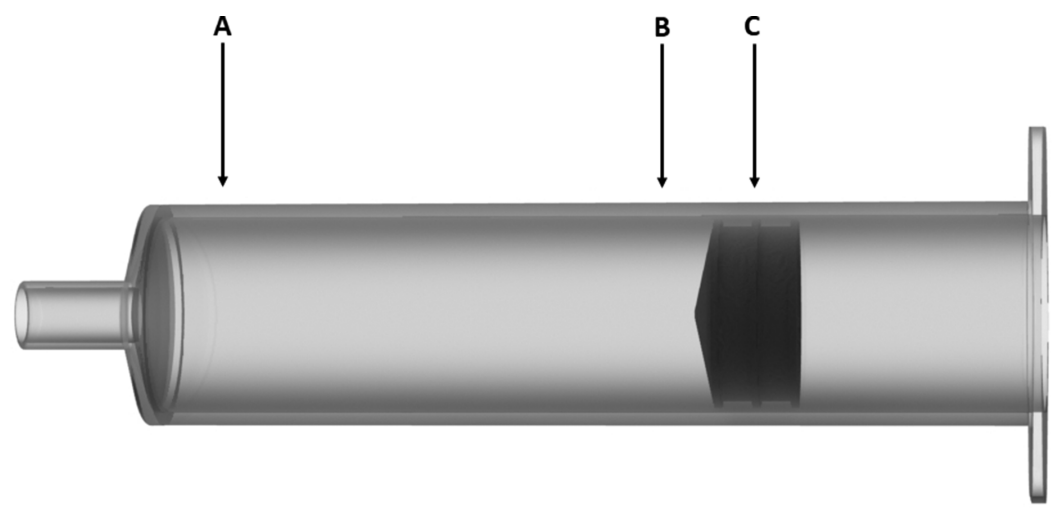

Figure 2: Positions of diameter measurement. 
Table 1: Results of the closure integrity test.

\begin{tabular}{lrr}
\hline Syringe type A & & \\
\hline & $\begin{array}{r}\text { Sterilized } \\
(\mathrm{n}=10)\end{array}$ & $\begin{array}{r}\text { Not sterilized } \\
(\mathrm{n}=2)\end{array}$ \\
\hline $\begin{array}{c}\text { Closure integrity visual inspection } \\
\text { (\% syringes with dye present) }\end{array}$ & $0 \%$ & $0 \%$ \\
$\begin{array}{c}\text { Closure integrity UV-Vis absorption } \\
\text { (\% syringes with dye present) }\end{array}$ & $0 \%$ & $0 \%$ \\
\hline
\end{tabular}

\section{Syringe type B}

\begin{tabular}{lrr}
\hline & $\begin{array}{r}\text { Sterilized } \\
(\mathrm{n}=10)\end{array}$ & $\begin{array}{r}\text { Not sterilized } \\
(\mathrm{n}=2)\end{array}$ \\
\hline $\begin{array}{c}\text { Closure integrity visual inspection } \\
\text { (\% syringes with dye present) }\end{array}$ & $0 \%$ & $0 \%$ \\
$\begin{array}{c}\text { Closure integrity UV-Vis absorption } \\
(\% \text { syringes with dye present) }\end{array}$ & $0 \%$ & $0 \%$ \\
\hline
\end{tabular}

caused by the liquid and gas separately and Figure 3(E) provides these results combined. In Figure $3(\mathrm{~F})$ the total volume change is converted to total stopper movement in millimeters for both brands. The total stopper movement for brand A using the data from Pt100 sensor I and II is, respectively 1.29 and $1.27 \mathrm{~mm}$. The total stopper movement for brand $\mathrm{B}$ using the data from Pt100 sensor I and II was 1.42 and $1.40 \mathrm{~mm}$, respectively.

\section{Sterility testing}

No microbiological growth was observed in the syringes for both brand A and B following incubation at $30-35^{\circ} \mathrm{C}$ for 14 days.
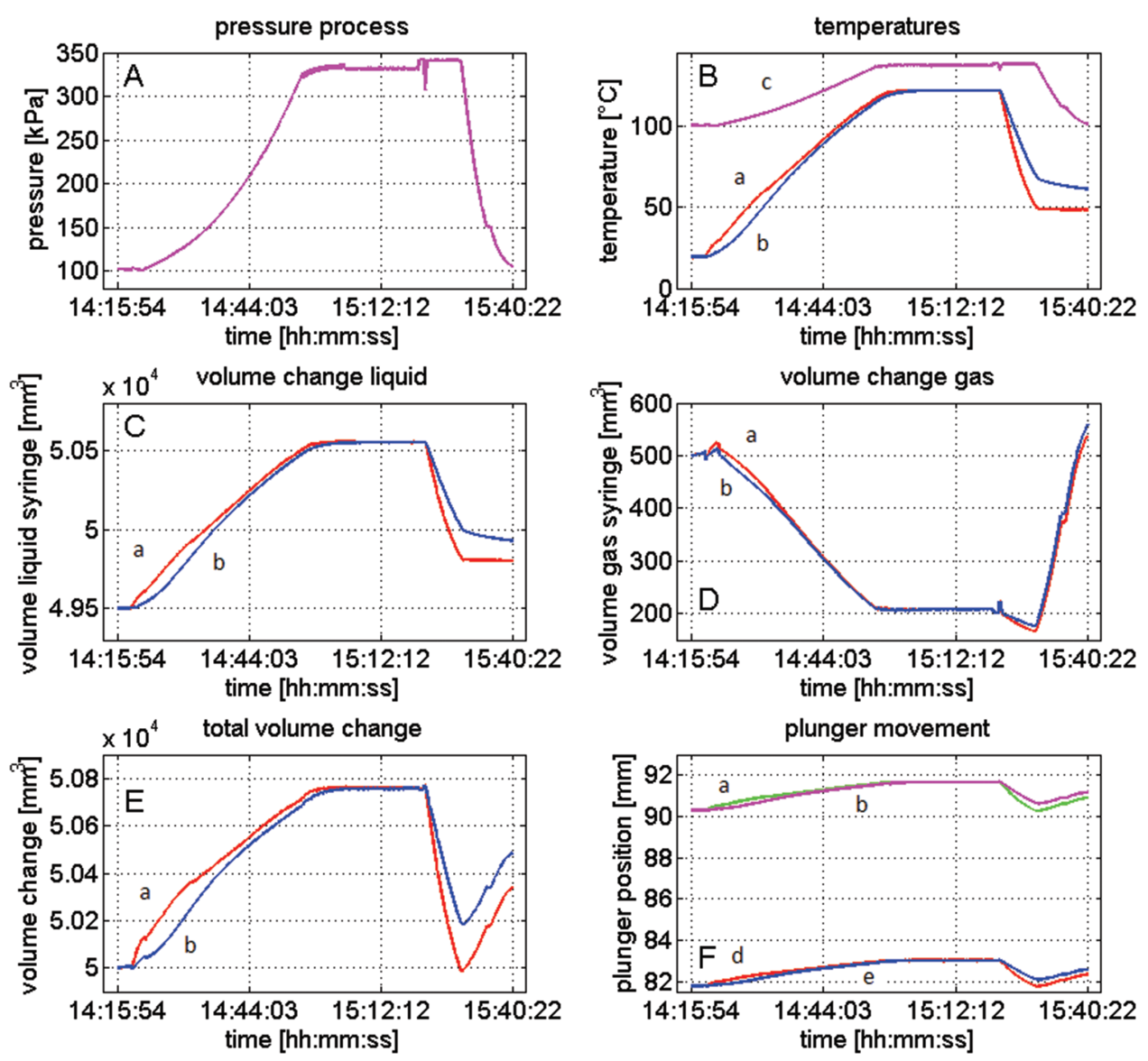

Figure 3: Results calculation stopper movement. Figure 3(A) depicts the pressure during the process. Figure 3(B) depicts the temperature during the process, where curve a and b depict the results from the two pt100 sensors and curve c depicts the temperature in the chamber. Figure 3(C-E) depict the liquid, gas and combined volume change calculated using the two pt100 sensors (curve a and b). Figure 3 (F) curve $a$ and $b$ depict plunger movement for brand $A$ and curve $d$ and e depict plunger movement for brand $B$ calculated with the results from the two pt100 sensors. 


\section{Other physical tests}

The results for weight, diameter and physical appearance are shown in Table 2. Weight and diameter for both brands appeared not normally distributed, therefore the Wilcoxon rank test was performed. There was a very minimal, although significant difference in median (range) weight before (80.54 $\mathrm{g}(80.06-81.50))$ and after (80.55 g (80.07-81.51)) sterilization for syringe brand A $(\mathrm{Z}=2.070, p<0.05)$. No weight difference was found for syringe brand $\mathrm{B}(79.05 \mathrm{~g}(78.78-79.31))$ and after (79.05 g (78.78-79.31) $(\mathrm{Z}=1.414, \mathrm{p}=0.157)$. There was no significant difference in diameter at the three points of measurement before and after sterilization for syringe brand $\mathrm{A}$ and $\mathrm{B}$. In addition, no physical deformation of the syringe, tipcap and stopper was observed after sterilization. Furthermore, no change in color and clarity had occurred due to sterilization.

\section{Discussion}

This study shows that the physical stability of prefilled syringes is guaranteed following water cascade sterilization. Only small deviations in weight before and after sterilization were found in ten out of twenty syringes. Although the statistical analysis indicated a significant increase in weight after sterilization for syringe brand A, the increase in weight was only minimal $(0.01 \mathrm{~g})$. A likely explanation for this slight increase in weight could be that the syringes measured before sterilization were completely dry, whereas the syringes measured after sterilization were not yet completely dry on the outside, only not visible to the naked eye. The fact that no penetration of liquid had occurred during sterilization is further supported by the fact that the closure integrity test showed no penetration of methylene blue. There was no significant difference in weight before and after sterilization for syringe brand B. The time between sterilization and weight measurement after sterilization was several days longer for brand $B$ than that for brand $A$, supporting the claim that the syringes of brand $A$ were measured before they were completely dry. The results for brand B indicate that no product evaporated out of the syringe during sterilization and no substance has penetrated the syringe from outside. The penetration of dye between the ribs of the stopper with the closure integrity test can be explained by the use of a needle to de-aerate the syringe. The needle could have influenced the integrity of the stopper and therefore dye could penetrate between the ribs.

The sterilization program used is validated for flask sterilization and not syringe sterilization. The syringes were sterilized with a full load of $100 \mathrm{ml}$ vials. However, it is likely that the heat distribution inside the sterilizer

Table 2: Results of weight and diameter comparison before and after sterilization and visual inspection.

\begin{tabular}{|c|c|c|c|}
\hline \multicolumn{4}{|l|}{ Syringe type A } \\
\hline & Before sterilization & After sterilization & $p$-value \\
\hline Weight $(\mathrm{g})(\mathrm{n}=10)$ & $80.54 \pm 1.44 ; 80.57$ & $80.55 \pm 1.44 ; 80.57$ & $<0.05$ \\
\hline Diameter A $(\mathrm{mm})(\mathrm{n}=5)$ & $31.00 \pm 0.25 ; 31.02$ & $30.95 \pm 0.20 ; 30.98$ & 0.28 \\
\hline Diameter B $(\mathrm{mm})(\mathrm{n}=5)$ & $31.20 \pm 0.20 ; 31.22$ & $31.20 \pm 0.10 ; 31.24$ & 0.32 \\
\hline Diameter C $(\mathrm{mm})(\mathrm{n}=5)$ & $31.40 \pm 0.10 ; 31.36$ & $31.30 \pm 0.10 ; 31.33$ & 0.47 \\
\hline Visual inspection ( $\%$ anomalies) $(n=10)$ & NA & $0 \%$ & NA \\
\hline \multicolumn{4}{|l|}{ Syringe type B } \\
\hline & Before sterilization & After sterilization & $p$-value \\
\hline Weight $(\mathrm{g})(\mathrm{n}=10)$ & $79.05 \pm 0.53 ; 79.01$ & $79.05 \pm 0.53 ; 79.01$ & 0.16 \\
\hline Diameter A $(\mathrm{mm})(\mathrm{n}=5)$ & $29.30 \pm 0 ; 29.30$ & $29.30 \pm 0 ; 29.30$ & 1 \\
\hline Diameter B $(\mathrm{mm})(\mathrm{n}=5)$ & $29.40 \pm 0.05 ; 29.39$ & $29.40 \pm 0.1 ; 29.42$ & 0.18 \\
\hline Diameter C $(\mathrm{mm})(\mathrm{n}=5)$ & $29.50 \pm 0.05 ; 29.48$ & $29.55 \pm 0.1 ; 29.55$ & 0.10 \\
\hline Visual inspection ( $\%$ anomalies) $(n=10)$ & NA & $0 \%$ & NA \\
\hline
\end{tabular}

Results, if not otherwise stated, are expressed as median \pm range; mean. NA: Not Applicable. 
will differ from that of a full load of $50 \mathrm{ml}$ syringes. The sterilization process is regulated by measuring the temperature in the glass vials. The vials contain a similar aqueous solution to the solution inside the syringes, therefore the heat conductivity of the solution inside the vials and syringes is similar. Heat conductivity of glass is larger than that of $\mathrm{COC}$ and COP. However, the syringes have a smaller volume and larger surface area that is in contact with the hot water inside the sterilizer compared to the volume and surface area of the vials, resulting in a faster warm-up of the liquid inside the syringe. We assume that these two variables outweigh the lower heat conductivity of $\mathrm{COC}$, and thus the temperature inside the syringes is the same as the temperature measured inside the vials. Therefore, this process indicates an adequate sterilization process for the $50 \mathrm{~mL}$ syringes. When further implementing this method a full validation with temperature measurement inside the syringe still needs to be performed to ascertain an adequate sterilization cycle.

The sterility testing with TSB showed that the microbiological stability is assured.

The maximum stopper movement calculated for brand $A$ and B are respectively 1.29 and $1.42 \mathrm{~mm}$. This distance is much less than the height of the stopper $(12 \mathrm{~mm})$, therefore the content of the syringe never comes into contact with the non-sterile part of the syringe [7].

In the current USP it is stated that closure integrity testing can replace sterility tests in stability studies and the dye ingress test as performed in this study is an accepted alternative $[14,15]$. Together with the results for weight measurements, stopper movement and sterility testing these results suggest that microbiological stability is guaranteed during sterilization. To assure microbiological stability during shelf-life physical dye ingress testing or sterility testing during shelf-life should be investigated.

No significant change in diameter occurred after sterilization and there is no deformation of the syringe at the height of the stopper, for both brands. All results indicate that no significant changes occur to the syringe, tipcap and stopper following sterilization, therefore the physical stability is guaranteed.

\section{Conclusion}

The results of this study demonstrate the proof of concept that the physical and microbiological stability of the cyclic olefin (co)polymer syringes is guaranteed during sterilization using a water cascade sterilizer. These results do not rule out the necessity of further chemical stability experiments with individual drug products in prefilled syringes and the validation of the process with a full load of syringes. These tests are necessary to fully validate the process of water cascade sterilization as a suitable and valid method for the sterilization of prefilled syringes.

Conflict of interest statement: The authors state no conflict of interest. The authors have read the journal's Publication ethics and publication malpractice statement available at the journal's website and hereby confirm that they comply with all its parts applicable to the present scientific work.

\section{References}

1. Suvikas-Peltonen E, Hakoinen S, Celikkayalar E, Laaksonen R, Airaksinen $M$. Incorrect aseptic techniques in medicine preparation and recommendations for safer practices: a systematic review. Eur J Hosp Pharm 2017;24:175-81.

2. Scheepers H, Beaney A, Le Brun P, Neerup Handlos V, Schutjens M, Walser S, et al. Aseptic preparation of parenteral medicinal products in healthcare establishments in Europe. Eur J Hosp Pharm 2016;23:50-3.

3. Van Grafhorst JP, Foudraine NA, Nooteboom F, Crombach WHJ, Oldenhof NJJ, Van Doorne H. Unexpected high risk of contamination with staphylococci species attributable to standard preparation of syringes for continuous intravenous drug administration in a simulation model in intensive care units. Crit Care Med 2002;30:833-6.

4. Rashed AN, Tomlin S. Sources and magnitude of error in preparing morphine infusions for nurse-patient controlled analgesia in a UK paediatric hospital. Int J Clin Pharm 2016;38:1345-1345.

5. Parshuram CS, To T, Seto W, Trope A, Koren G, Laupacis A. Systematic evaluation of errors occurring during the preparation of intravenous medication. Can Med Assoc J 2008;178:42-8.

6. McDowell SE, Mt-Isa S, Ashby D, Ferner RE. Where errors occur in the preparation and administration of intravenous medicines: a systematic review and Bayesian analysis. Qual Saf Heal Care 2010;19:341-5.

7. Harrison B, Rios M. Big shot: developments in prefilled syringes. Pharm Technol 2007;31:50-60.

8. Dierick W. Ready-to-use prefillable syringes: sterilisation effects on biopharmaceuticals. Drug Deliv 2016;71:50-4.

9. Nishimoto N, Maekawa T. Determination of conditions for the production scale sterilization of prefilled syringes. PDA J Pharm Sci Technol 2003;57:376-86. 
10. Parenteral Drug Association P. PDA Technical Report No. 48 (TR 48) Moist Heat Sterilizer Systems: Design, Commissioning, Operation, Qualification and Maintenance. 2010.

11. Bouwman-Boer Y, Fenton-May V, Le Brun P, editors. Practical Pharmaceutics - An International Guideline for the Preparation Care and Use of Medicinal Products: Springer International Publishing, Cham, Switzerland, 2015:680-84 p.

12. International Organization for Standardization. Sterilization of health care products - Moist heat - Part 1: requirements for the development, validation and routine control of a sterilization process for medical devices (ISO 17665-1) [Internet]. 2006.
Available from: https://www.iso.org/standard/43187.html. Cited 2017 Jun 14.

13. Parenteral Drug Association P. PDA Technical Report No. 27 (TR 27) Pharmaceutical Package Integrity. 1998.

14. FDA. Guidance for industry container and closure system integrity testing in lieu of sterility testing as a component of the stability protocol for sterile products [Internet]. 2008. Available from: http://www.fda.gov/cber/guidelines.htm. Cited 2017 Jun 14.

15. ICH. Q5C stability testing of biotechnological/biological products. ICH Harmonised Tripartite Guideline. 1995. p. 1-8. 